\section{COGNITIVE CONTROL}

\section{“One Moment, Let Me Just Get This Text ..."}

OPHIR ET AL. (2009). Cognitive control in media multitaskers. PNAS, 106, 15583.

Recent technologies allow users to be online at all times through monitoring their phones, text messages, e-mails, and Web sites, often simultaneously. Ophir et al. investigated the consequences of this "media multitasking" on the control of basic cognitive processes. Media multitasking has two potential effects: On the one hand, directing attention and other limited cognitive resources from one environmental source to another might reduce the typical limitations of human cognition through mere practice, allowing cognition to be more flexible and reducing the costs of performing several tasks simultaneously. On the other, media multitasking might make cognitive control more difficult.

To investigate these issues, Ophir et al. used an individual differences approach, increasingly popular in the study of cognition (e.g., Vogel \& Awh, 2008, Curr Dir Psychol Sci 17:171). First, Ophir et al. developed a questionnaire to identify individuals who were heavy versus light media multitaskers. These individuals then performed several lab-based cognitive tasks that tapped specific cognitive processes. Perhaps the most relevant was task switching, in which participants reported either whether a number was even or odd or whether a letter was a consonant or vowel. The two tasks were intermixed, and task switching was indexed by examining the reaction time on the current task as a function of the previous trial's task. Typically, participants are slower when the task switches from trial $n-1$ to trial $n$ than when the task remains the same between trials. Heavy media multitaskers exhibited a larger task switch effect than did light multitask- ers, and similar results occurred for other cognitive tasks. For example, in both a visual memory task and a continuous performance task, the number of task-irrelevant distractors produced a greater memory decrement in heavy than in light media multitaskers. Interestingly, there were few, if any, differences in results for the latter two tasks when distractors were absent.

Ophir et al.'s results are provocative in suggesting that directing limited processes to multiple sources does not improve such multitasking behavior as task switching. Although the findings are correlational, one straightforward and testable explanation of them is that various technologies may emphasize the bottom-up control of attention and cognition. Heavy media multitaskers may relinquish top-down control, thereby becoming more susceptible to the effects of distractors and becoming hindered when top-down control must be exercised, as in task switching. - S.P.V.

\section{VISUAL PERCEPTION}

\section{Perceiving Surface \\ Properties}

ANDERSON \& Kim (2009). J Vis, 9(11), Art. 10.

Human vision is able to make subtle judgments about natural surfaces. For example, we easily judge whether a rock face is likely to hold our boot without slipping as we hike, or whether a sponge is moist or dry. A lively debate has recently sprung up over the question of how we make such judgments; in general, the problem of determining the properties (e.g., the albedo, reflectance, and depth variations) of a surface from the pattern of light it reflects is very complicated. In 2007, Motoyoshi et al. (Nature 447:206) presented evidence that vision may not extract these properties at all, but instead may use surprising computational "shortcuts" in estimating them. Specifically, the researchers used photographs of bumpy, materially homogeneous surfaces and showed that if one fixes the mean luminance of such an image, the perceived albedo of the surface is sensitively controlled by the skew of the image's gray-level histogram. Positive skew makes the surface look glossy; negative skew makes it look matte. The spatial pattern of light reflected by the surface is irrelevant to this statistic: You can scramble the pixels in the image without altering the skew of the histogram, so if skew really does control our visual judgments of surface albedo, this is a serious shortcut indeed. Anderson and Kim have mounted a concerted assault on this claim. In essence, they argue that Motoyoshi et al.'s results are an artifact of the restricted set of surfaces they used. When you fix the mean of an image of a materially homogeneous surface and massage the histogram to alter its skew, the only possible effects, Anderson and Kim argue, are the following: Making the skew positive turns the brightest points of the image into specular highlights, so the surface looks glossy; making the skew negative sharpens the depths of shaded regions but, more importantly, strips away the highlights so the surface looks matte. Anderson and Kim presented a range of different surfaces for which altering the skew of the histogram had no influence on the perceived albedo. This will likely not be the last word in the debate, so stay tuned. - C.F.C.

\section{AUDITORY PERCEPTION}

\section{What Each \\ Hemisphere Hears}

González \& McClennnan (2009). Hemispheric differences in the recognition of environmental sounds. Psychol Sci, 20, 887.

Given the dizzying complexity of our perceptual world, perceivers must track specific aspects of the environment as well as categories of objects more generally. Depending on the sit- 
uation, we may, for example, identify the nuanced toot of our grandmother's car horn signaling that she has arrived, or simply classify the sound as a car horn and get out of the way. Research examining hemispheric processing differences in visual object recognition (Marsolek, 1999, Psychol Sci 10:111) has led to the proposal that separate and dissociable neural subsystems for specific versus abstract processing are associated with the right versus the left hemisphere. González and McClennan have found that the relative specificity of hemispheric processing extends beyond visual object and spoken word recognition to environmental sounds.

Across four experiments, their participants were presented with environmental sounds, such as thunder, tooth brushing, an elephant, and ping pong, in a long-term repetition priming paradigm. From study to test, sounds were repeated in either the same or a different form and to the right or to the left ear. Specificity effects in priming were found for sounds presented to the left ear (right hemisphere), but not for sounds presented to the right ear (left hemisphere). Hemispheric differences in the processing of environmental sounds suggest that differential specificity effects crosscut particular modalities. This work lends support to the notion of a general neural architecture for perception, categorization, and memory that consists of dissociable neural subsystems for abstract and specific perceptual processing. - L.C.N.

\section{LANGUAGE AND THE BRAIN}

\section{Why Scientists Have Better Vocabularies}

BADDELEY \& ATteWELl (2009). The relationship between language and the environment. Psychol Sci, 20, 1100.

Baddeley and Attewell argue that descriptive terms are used with a frequency defined by their ability to reduce uncertainty. In particular, only three terms for lightness are used with any nonnegligible frequency, because natural stimuli are just under three times more variable than an observ- er's memory. Those words are white (or light), black (or dark), and gray. Of course, words like blinding and fuligin do exist, but they are used so infrequently that their reduction of uncertainty about surface reflectance is negligible. Prima facie, the "magic number" 3 seems at odds with Miller's (1956, Psychol Rev 63:81) famous argument for $7 \pm 2$, in which a previously presented stimulus could be correctly identified only when the choices were limited to $7 \pm 2$ similar stimuli with different magnitudes. However there really is no discrepancy. Miller's musings were based on laboratory experiments, which Baddeley and Attewell replicated. What Miller did not consider is how uncertainty can be reduced by the simple knowledge that natural stimuli are viewed outside the laboratory! Furthermore, because the homogeneity of reflectances can vary with viewing environment, different environments support different numbers of descriptive terms for lightness. For example, Baddeley and Attewell calculated that only two terms are necessary for the maximum reduction in uncertainty about woodland reflectances. It remains to be seen whether our use of language really changes when we describe samples from more and less homogeneous sets of stimuli. Perhaps we should look across dimensions (e.g., reflectance vs. size or age) instead of across environments for confirmation of Baddeley and Attewell's ideas. - J.A.S.

\section{TIME PERCEPTION}

\section{Spatio-Temporal Distortions}

Frassinetti et AL. (2009). Prismatic lenses shift time perception. Psychol Sci, 20, 949.

How sensitive is time perception to spatial factors? To study this question, Frassinetti et al. used a prismatic task in which participants were asked to point to a target at one of three positions (left, central, or right) on a horizontal plane while wearing or not wearing prismatic lenses. Participants were also asked to perform a time reproduction task in which they sought to reproduce the duration (or half the duration) of a target presented for 1.6-2.4 sec. This temporal task was performed before or after adaptation to the prismatic effect. The results revealed that altering spatial attention has a significant impact on temporal processing, and that the direction of this influence is related to the direction of the prismatic orientation: There is an underestimation of duration after leftward adaptation and an overestimation after rightward adaptation. This study demonstrates that the representation of temporal intervals is horizontally arranged in space and that spatial attention links time and space in a so-called generalized magnitude system. - S.G.

\section{VISUAL PROCESSING}

\section{The Locus of Crowding Effects}

Chakravarthi \& Cavanagh (2009). Recovery of a crowded object by masking the flankers: Determining the locus of feature integration. JVis, 9(10), Art. 4.

Where in the brain, in the course of visual processing, are local features put together in a manner that permits object recognition? One might expect that question to frame a story about the latest fMRI localization study. However, Chakravarthi and Cavanagh remind us that there are elegant behavioral/psychoanatomical methods for addressing such issues. In their experiment, observers tried to identify the orientation of a C-like figure surrounded by four other similar figures. These flanking Cs produced a "crowding" effect. When the flankers were present, an otherwise identifiable $\mathrm{C}$ became unidentifiable. Crowding is thought to occur when features from the flankers get pooled with features from the target, disrupting target identification. Chakravarthi and Cavanagh used several masking methods to attempt to kill off the features of the flankers before they have a chance to interfere with the central object. If crowding stimuli are followed by a superimposed noise pattern, the identification of those stimuli can be brought to near-chance levels. This sort of noise mask is thought to operate early in visual pro- 
cessing. When the flanking Cs were marked in this way, crowding of the central C was largely eliminated. In contrast, an object substitution mask did not interfere with crowding. The object substitution mask was just as effective as the noise masking in reducing identification of the flanking masks to chance levels. However, by the time the object substitution mask suppressed the features of the flanking items, those features had already produced a crowding effect. Thus, the locus of crowding lies after noise masking but before object substitution. In addition, if you flash a circle around flanking letters with the correct timing, you can produce metacontrast masking. This is thought to occur after noise masking and before object substitution, and indeed it did produce more of a release from crowding than did object substitution, but less than noise masking. Chakravarthi and Cavanagh argue that these results, combined with those of other behavioral and neuroimaging studies, point to a location for crowding that comes after primary visual cortex and before lateral occipital cortex. - J.M.W.

\section{TASTE PERCEPTION}

\section{Fizzy Buds}

ChandrasheKar ET AL. (2009). The taste of carbonation. Science, 326, 443.

When people are thirsty, they often reach for a soft drink to quench their thirst. We like the taste of soft drinks, and soft drink companies spend billions of dollars each year advertising the unique and refreshing tastes of their products. One ingredient that is often left out of these discussions, but which is common to all, is carbonation. Carbonation can be sensed in a variety of ways by our bodies; soft drinks are enjoyable, in part, because we can feel the bursting bubbles on our tongues and in our mouths. But, recent evidence provided by Chandrashekar et al. now reveals that carbonation can also be sensed by the taste receptors on the tongue. As is well known, human taste receptors fall into five categories: bitter, sweet, salty, sour, and unami. In an elegant series of experiments using both normal and genetically altered mice, Chandrashekar et al. showed that the taste of carbonation is conveyed specifically by the sour receptors. In their first set of experiments, these researchers monitored electrophysiological responses to a wide variety of stimuli in the chorda tympani, which is one of the major cranial nerves that carries taste information from the tongue to the brain. As expected, the results showed activation by well-known stimuli such as acesulfame K (sweet), quinine (bitter), sodium chloride (salty), and citric acid (sour). However, in addition, activation was also observed when club soda and carbon dioxide $\left(\mathrm{CO}_{2}\right)$ were presented on the tongue, and this ac- tivation was found to be dose dependent. Next, Chandrashekar et al. measured electrophysiological activity in mice that had been engineered to be missing one of the five receptors. The findings showed that $\mathrm{CO}_{2}$ produced activation in all of the mice except those that lacked the sour receptors. Digging deeper to understand how $\mathrm{CO}_{2}$ activates the taste system, the researchers targeted a gene-known as Car4 - that expresses sour receptors. The Car4 gene encodes the carbonic anhydrase 4 enzyme, which is responsible for converting $\mathrm{CO}_{2}$ into bicarbonate ions and free protons. Two more experiments were conducted to implicate these enzymes: one in which engineered mice lacked these enzymes, and one in which benzolamide was injected into normal mice to inhibit the action of the enzymes. As predicted, the results showed a reduced response to $\mathrm{CO}_{2}$ in both groups of mice. Interestingly, although the response to $\mathrm{CO}_{2}$ was nearly abolished in the two groups of mice, the response to other sour tastes such as citric acid was normal, suggesting that carbonic anhydrase is the primary $\mathrm{CO}_{2}$ receptor in the sour system. But why did mammals evolve a specific receptor for $\mathrm{CO}_{2}$ ? The researchers speculate that this receptor system may have evolved to detect the presence of $\mathrm{CO}_{2}$ in food that had fermented or was otherwise spoiled. - B.S.G. 